\title{
Vibration Feedforward Compensation for Magnetically Suspended Control and Sensitive Gyroscope with Spherical Rotor
}

\author{
Qiang Liu $\mathbb{D},{ }^{1}$ Heng Li, ${ }^{1}$ Cong Peng, ${ }^{2}$ Sha Sheng, ${ }^{1}$ Zhaojing Yin, ${ }^{1}$ and Zhiguo Liu ${ }^{1}$ \\ ${ }^{1}$ Institute of Precision Electromagnetic Equipment and Advanced Measurement Technology, \\ Beijing Institute of Petrochemical Technology, Beijing, China \\ ${ }^{2}$ College of Automation Engineering, Nanjing University of Aeronautics and Astronautics, Nanjing, Jiangsu, China \\ Correspondence should be addressed to Qiang Liu; liuqiangbuaa@163.com
}

Received 5 February 2020; Revised 17 September 2020; Accepted 24 October 2020; Published 6 November 2020

Academic Editor: Andrea Spaggiari

Copyright (C) 2020 Qiang Liu et al. This is an open access article distributed under the Creative Commons Attribution License, which permits unrestricted use, distribution, and reproduction in any medium, provided the original work is properly cited.

\begin{abstract}
Magnetically suspended control and sensitive gyroscope (MSCSG) with two capabilities including attitude control and attitude measurement is promising in meeting the requirements of novel spacecraft. When the momentum vector of a high-speed rotor suspended in five directions is tilted by Lorentz force-type magnetic bearing, the instantaneous large control moment is obtained. The spacecraft attitude can be sensed by a high-speed rotor without deflection interference torque. In this paper, a novel MSCSG with sphere rotor is presented, and its spherical structure and working principle are introduced. The magnetic bearing-rotor dynamic model is established based on Newton's second law and the gyrokinetic equations. The generating mechanism of three interferences, including bearing dynamic reaction force, deflection torque, and centrifugal force, is analyzed. The feedforward compensation control against three interferences for MSCSG is adopted, and the loading sequence of three interferences is simulated. The optimal sequence of bearing dynamic reaction force, deflection torque, and centrifugal force is used. The radial and axial vibration displacement amplitudes are reduced from $20.8 \mu \mathrm{m}$ and $31.7 \mu \mathrm{m}$ to $9.4 \mu \mathrm{m}$ and $14.9 \mu \mathrm{m}$, respectively. The experimental results are in good agreement with the simulation, which indicates the MSCSG rotor unbalance vibration is suppressed effectively by the feedforward compensation method.
\end{abstract}

\section{Introduction}

The ball bearing flywheels have been used in orbiting spacecraft for attitude control for several decades. Nevertheless, the metal-to-metal contact of the bearing elements and the need for the lubricant supply are unavoidable $[1,2]$. The emergence of magnetic bearings provides the possibility of solving this intractable problem. The use of magnetic bearing enables the flywheel to possess the characteristics of long service life, no stiction-friction effect, high reliability, and control precision [3-5]. Magnetically suspended momentum and reaction flywheels were applied in the SPOT satellites provided by Alcatel Space Industries $[6,7]$. The attitude control and energy storage flywheels suspended by magnetic bearings with high-speed rotors were used in the American Hubble Space Telescope [8]. The three types of inertial flywheels above are suitable for stability attitude control because of the high precision output torque [6-8].
However, the spacecraft is short of rapid manoeuvrability due to the little control torques above. The momentum vector of gimballing flywheels suspended in five directions can be tilted actively with respect to the spacecraft body to generate instantaneously large control torque. Thus, the magnetically suspended gimballing flywheel is promising in meeting the requirements of both precision and manoeuvrability $[9,10]$. When the deflection interference torque of the high-speed free suspension rotor is compensated by tilting magnetic bearings, the spacecraft attitude can be sensed by the rotor [11]. The two capabilities, attitude control and attitude measurement, are integrated in the magnetically suspended control and sensitive gyroscope (MSCSG).

The synchronous vibration in a magnetically suspended system is caused by the mass unbalance which resulted from manufacture errors and raw material mass unevenness $[12,13]$. The vibration interferences can be classified into 
three cases, the centrifugal force, the unbalanced mass moment, and the composite interference derived from the former two cases. The centrifugal force interference is caused by the parallel offset between the rotor mass centroid and the rotor rotating axis. To suppress the vibration induced by the centrifugal force, lots of feedback control methods are proposed by scholars. Takeshi analyzed the basic characteristics of the magnetically suspended control system based on the transfer function and introduced an active vibration control feedback compensation method [14]. There are considerable errors and variations of the power amplifier in the magnetically suspended control system when the temperature changes largely. To eliminate the errors and variations in [14], Fang et al. proposed an adaptive feedback compensation method with a gain phase modifier for the vibration in a magnetically suspended control moment gyroscope system [15]. The feedforward compensation method with a very high response speed compared with the feedback method is usually used for the magnetically suspended system. Jiang et al. proposed a feedforward compensation method based on the Fourier coefficients [16]. Shi et al. developed an adaptive feedforward compensation method based on the filtered- $x$ leased mean square algorithm for synchronous disturbance attenuation [17]. Both the feedforward compensation methods in $[16,17]$ are applied to suppress the unbalanced vibration of the rotor with a low speed of $3000 \mathrm{r} / \mathrm{min}$ and $1300 \mathrm{r} / \mathrm{min}$, respectively. Betschon and Knospe and Gao, respectively, proposed an adaptive feedforward control based on gain matrix function and a variable step size least mean square feedforward compensation strategy for suppressing the high-speed rotor vibration induced by the centrifugal force $[18,19]$. However, the influence of low-pass characteristics of the power amplifiers on vibration compensation accuracy in the control systems is not considered in [18, 19]. Wei and Xiang designed an adaptive feedforward compensation controller based on the LMS algorithm for the unbalance vibration control of magnetically suspended control moment gyroscope [20]. In addition, Zheng and Feng proposed a feedforward compensation method based on an adaptive notch filter used for suppressing the unbalance vibration of magnetically suspended compressor [21].

Due to the existence of the centrifugal force and the unbalanced mass moment, composite interference should be considered. Tang et al. and Xu et al. presented two feedback control methods using general notch filter and plural notch filter for suppressing the unbalance vibration of magnetically suspended flywheel and control moment gyroscope system, respectively $[22,23]$. Because of the narrow range of the trapping parameters of the notch filter in $[22,23]$, the compensation ability on the vibration of different frequencies is limited. Chen et al. presented a double-loop vibration compensation method for the rotor unbalance vibration of the magnetically suspended motor [24]. The interference coupling problem in the single loop is remedied by the double-loop without adopting the notch filter and setting fixed parameters.

The centrifugal force can be ignored when the rotor mass centroid lies in the rotor rotating axis. So, the unbalance mass moment is the main interference. Tang and Chen established the unbalanced vibration control model of the magnetically suspended control moment gyroscope and realized unbalanced vibration feedback control by combining the notch filter and the adaptive controller [25]. Bi et al. used the domain iterative learning algorithm to identify the unbalance distribution, and the unbalance vibration is compensated by the synchronous compensation signal [26]. The compensation methods for unbalance vibration in $[25,26]$ are verified by simulation. Li et al. adopted the general notch filter to suppress the same frequency current and used the feedback control to adjust the control current for suppressing the unbalance vibration [27]. However, the nonlinearity in the magnetic bearing system on unbalance vibration is not considered in [27]. Grochmal and Lynch and Tung et al. designed a nonlinear reduced-order disturbance observer and an integral type disturbance observer to estimate the unbalance mass moment and used nonlinear control and fuzzy gain tuner to compensate the unbalance vibration, respectively $[28,29]$. Due to the low estimation accuracy of the disturbance observer and the low stability of the control system at the high speed, both the precision and bandwidth of the disturbance observer should be considered simultaneously. Therefore, Gerlach et al. designed a selflearning adaptive filter to compensate the unbalance vibration of a high-speed rotor of the five-degree-of-freedom full-active high torque flywheel [30]. Since the high response speed of feedforward, Hui et al. and Zhou and Shi proposed two feedforward compensation methods [31, 32]. Hui et al. employed an adaptive feedforward control method to suppress the unbalance vibration of a magnetically suspended compressor [31]. Zhou and Shi designed a timevarying disturbance observer for estimating the unbalance mass moment of a magnetically suspended rigid rotor [32].

The conventional magnetic bearing air gap shapes with a cylindrical shell, thin wall, and cone shell will be changed when the rotor is driven from the equilibrium position. The interference torque is induced due to the gradient of the air gap magnetic flux density, and the rotor control accuracy is reduced. Therefore, the sphere magnetic bearings with a constant air gap are adopted for the MSCSG. When the sphere centroids of stator and rotor of sphere magnetic bearings coincide, the interference torque is suppressed and the rotor suspension accuracy is improved. The bearing dynamic reaction force and the centrifugal force are induced by the unbalance mass moment and the offset between the rotor mass centroid and the rotor rotating axis, respectively. In addition, the deflection torque around the rotor mass centroid is caused by the electromagnetic levitation force through the rotor sphere centroid, due to the offset between the rotor mass centroid and the rotor sphere centroid. Based on the analysis above [14-32], the bearing dynamic reaction force and the centrifugal force can be effectively suppressed. However, the influence of deflection torque and different loading sequence on the vibration suppression effect is not considered. In this paper, a novel MSCSG is presented, and its structure and working principle are introduced. The dynamic model of a magnetically suspended system is established. The feedforward compensation control against three interferences, bearing dynamic reaction force, 
deflection torque, and centrifugal force, is proposed. The experimental validations are provided with the control platform of the MSCSG prototype.

\section{Structure and Working Principle}

The setup of MSCSG is shown in Figure 1. The gyro rotor with the angular momentum of $15 \mathrm{Nms}$ is supported by the radial and axial magnetic bearings in three translation directions and the Lorentz force-type magnetic bearing in two radial deflection directions. The radial displacements are measured by the radial displacement sensors. The axial displacement sensors are utilized for measuring the axial displacement and the deflection angles. The gyro rotor is driven by a motor at a rated speed of $8000 \mathrm{r} / \mathrm{min}$ in a vacuum environment provided by gyro house and sealed cowlings. When the rotor supported by magnetic bearings with plane or cylindrical poles deviates from the equilibrium position, the air gap uniformity is changed. The disturbance deflection torque will be induced by the uneven suspension forces in magnetic poles. Therefore, the spherical magnetic poles with the constant air gap shape are adopted. For convenience of spherical rotor manufacture, the rotor is divided into the outer rotary disk and inner rotary shaft. The sphere centroid of the outer rotary disk is located in the central axis of its inner cylindrical hole used for fitting with the inner rotary shaft. The upper and lower sphere centroids of the inner rotary shaft are coincided, and both of them locate in the central axis of its outer cylindrical surface. The three-sphere centroids of the outer rotary disk and inner rotary shaft coincide by means of adjusting the thickness of the adjustable ring. The structure and working principle have been described in detail in $[9,10]$.

\section{Magnetic Bearing-Rotor Dynamics Model}

The coordinate system of MSCSG is shown in Figure 2. The coordinate system OXYZ of magnetic bearing-rotor is established. $O$ is the coordinate system origin coinciding with the rotor sphere centroid. The $z$-axis of the coordinate system is in coincidence with the rotor geometric axis. The $x$ and $y$-axes are defined according to the right hand rule. $O_{I}$ is the rotor mass centroid. $\omega$ is the rotary speed of rotor. $R$ is the installation radius of the Lorenz force-type magnetic bearing coils. $\alpha$ and $\beta$ are the deflection angles of the rotor around the $x$-axis and the $y$-axis. $l_{r}$ and $l_{a}$ are the distances between the displacement sensors and the origin $O$ in the radial and axial directions. $l_{a n}$ and $l_{b n}$ are the distances between the two counterweight surfaces and XOY plane. $f_{x}$, $f_{y}$, and $f_{z}$ are the translation electromagnetic forces of the radial and axial magnetic bearings. $P_{x}$ and $P_{y}$ are the deflection torques of Lorentz force-type magnetic bearing. $f_{l}$ is the ampere force generated by single coil of the Lorenz forcetype magnetic bearing.

The simplified approach is suggested for control and simulation analysis of MSCSG. The magnetic bearing control system is linear time invariant. The sphere rotor deformation in high rotary speed and gravity effect on ground are ignored. The same power amplifiers of radial and axial translation suspension are used. Based on Newton's second law and the gyrokinetic equation [33], the rotor dynamics equations are established as follows:

$$
\left\{\begin{array}{l}
m \ddot{x}=f_{x}, \\
J_{y} \ddot{\beta}-J_{z} \omega \dot{\alpha}=p_{y}, \\
m \ddot{y}=f_{y}, \\
J_{x} \ddot{\alpha}+J_{z} \omega \dot{\beta}=p_{x}, \\
m \ddot{z}=f_{z} .
\end{array}\right.
$$

Equation (1) can be written as

$$
M \ddot{\mathrm{q}}+G \dot{\mathrm{q}}=F,
$$

where $\quad M=\left[\begin{array}{ccccc}m & 0 & 0 & 0 & 0 \\ 0 & J_{y} & 0 & 0 & 0 \\ 0 & 0 & m & 0 & 0 \\ 0 & 0 & 0 & J_{z} & 0 \\ 0 & 0 & 0 & 0 & m\end{array}\right], \quad \mathrm{q}=\left[\begin{array}{lllll}x & \beta & y & \alpha & z\end{array}\right]^{\mathrm{T}}, \quad G=$ $\left[\begin{array}{ccccc}0 & 0 & 0 & 0 & 0 \\ 0 & 0 & 0 & -1 & 0 \\ 0 & 0 & 0 & 0 & 0 \\ 0 & 1 & 0 & 0 & 0 \\ 0 & 0 & 0 & 0 & 0\end{array}\right] J_{z} \omega, \mathrm{F}=\left[\begin{array}{lllll}f_{x} & p_{y} & f_{y} & p_{x} & f_{z}\end{array}\right]^{\mathrm{T}}$ represent the mass matrix, the generalized coordinate, the gyroscopic matrix and generalized force, respectively, $m$ is the rotor mass, $J_{x}$ and $J_{y}$ are the transverse inertia moments of the rotor, and $J_{z}$ is the polar inertia moment of the rotor. The transfer function $\Phi(s)$ of the dynamics model can be described as

$$
\Phi(s)=\left(M s^{2}+G s\right)^{-1} .
$$

The generalized force can be linearized as follows:

$$
F=K_{h} q+K_{i} I
$$

where $\quad K_{h}=\left[\begin{array}{ccccc}k_{h x} & 0 & 0 & 0 & 0 \\ 0 & 0 & 0 & 0 & 0 \\ 0 & 0 & k_{h y} & 0 & 0 \\ 0 & 0 & 0 & 0 & 0 \\ 0 & 0 & 0 & 0 & k_{h z}\end{array}\right]$ and $K_{i}=$
$\left[\begin{array}{ccccc}k_{i x} & 0 & 0 & 0 & 0 \\ 0 & k_{i \beta} & 0 & 0 & 0 \\ 0 & 0 & k_{i y} & 0 & 0 \\ 0 & 0 & 0 & k_{i \alpha} & 0 \\ 0 & 0 & 0 & 0 & k_{i z}\end{array}\right]$ are the force-displacement stiffness

factor and the force-current stiffness factor, and $\boldsymbol{I}=\left[\begin{array}{lllll}i_{x} & i_{\beta} & i_{y} & i_{\alpha} & i_{z}\end{array}\right]^{\mathrm{T}}$ is the magnetic bearing currents. The control currents of the magnetic bearings under decentralized PID control law can be described as

$$
I=K_{s} K_{w}\left(-K_{P} q_{s}-K_{I} \int q_{s} \mathrm{~d} t-K_{D} \dot{\mathrm{q}}_{s}\right),
$$

where $\mathrm{K}_{\mathrm{s}}$ and $K_{w}$ are the gains of sensor and power amplifier and $\mathrm{K}_{\mathrm{P}}, \mathrm{K}_{\mathrm{I}}$, and $\mathrm{K}_{\mathrm{D}}$ are the proportion gain, integration gain, and differential coefficients. $\mathrm{q}_{\mathrm{s}}=\left[\begin{array}{lllll}x_{s} & \beta_{s} & y_{s} & \alpha_{s} & z_{s}\end{array}\right]^{\mathrm{T}}$ is the sensor coordinate. The deviation angle between the displacement sensors and radial magnetic bearing in the circumferential direction is $\pi / 4$. Therefore, the generalized coordinate q can be written as 


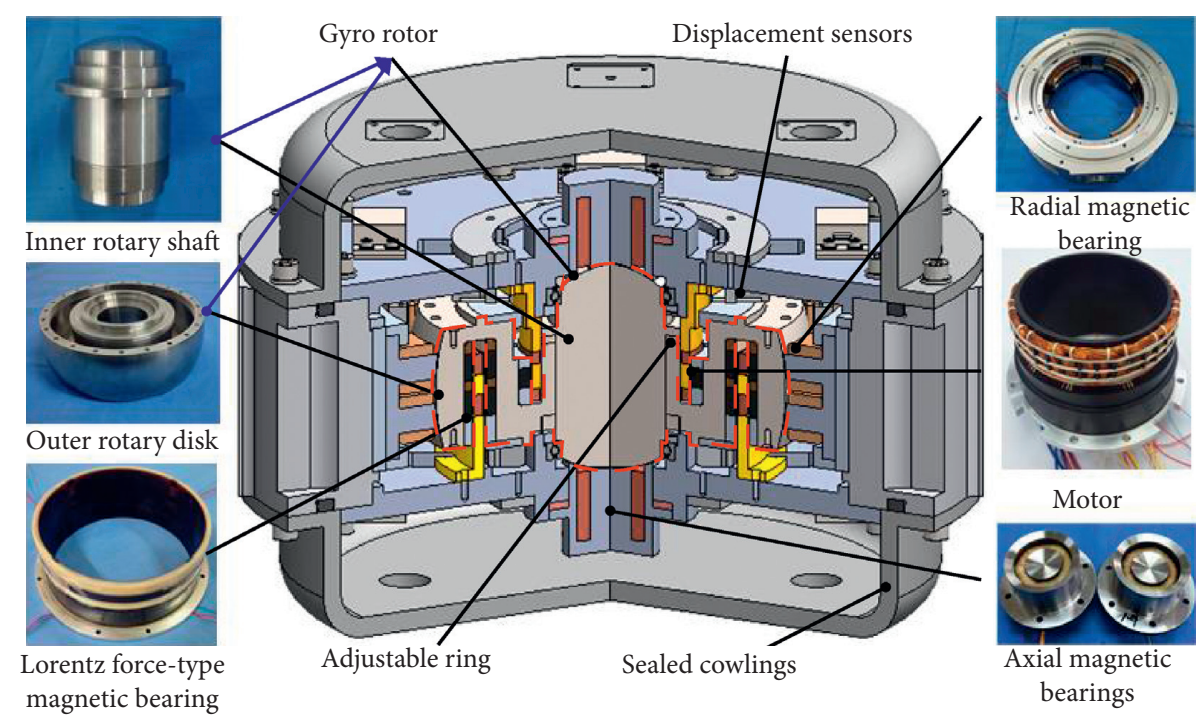

FIgURE 1: Configuration of MSCSG.

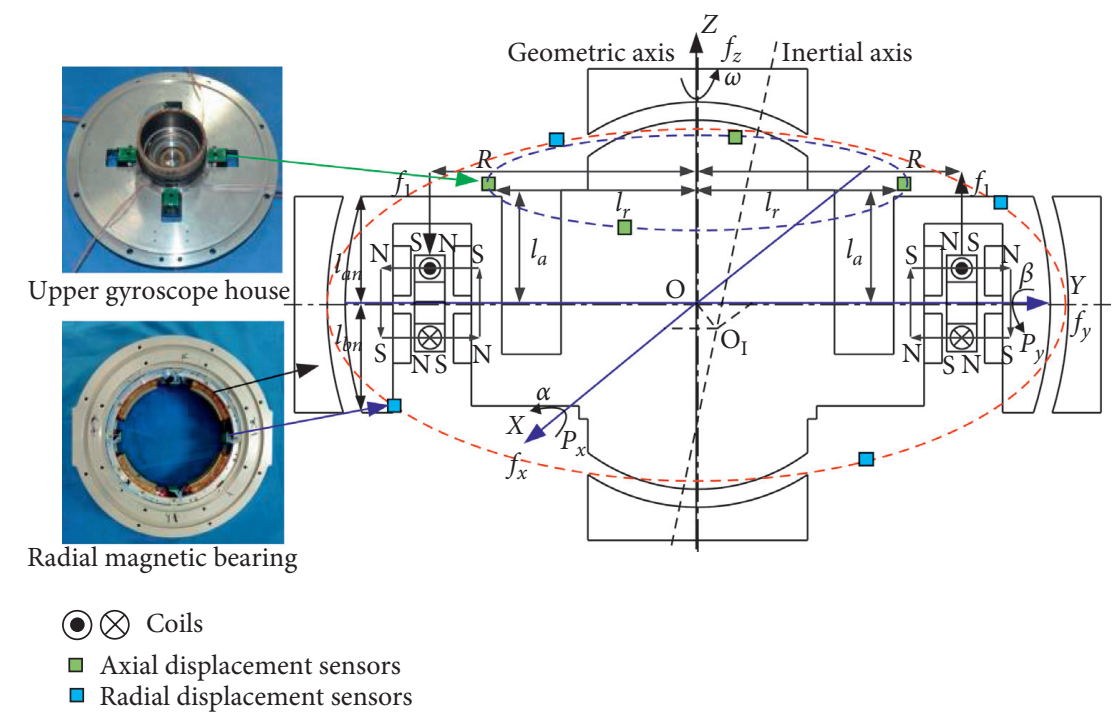

Figure 2: Coordinate system of MSCSG.

$q=\left[\begin{array}{ccccc}\cos \left(\frac{\pi}{4}\right) & 0 & 0 & 0 & 0 \\ 0 & 1 & 0 & 0 & 0 \\ 0 & 0 & \sin \left(\frac{\pi}{4}\right) & 0 & 0 \\ 0 & 0 & 0 & 1 & 0 \\ 0 & 0 & 0 & 0 & 1\end{array}\right] \cdot q_{s}$

(6)

\section{Generating Mechanism of Interferences}

No matter whether the rotor deflects, the shape of the sphere shell air gap is invariable in the condition of the coincidence of the stator and rotor sphere centroids. The deflection interference torque of sphere magnetic bearings is eliminated. Under this condition, there are only three interferences as follows:

(1) The bearing dynamic reaction force caused by a certain unbalance mass moment.

(2) The centrifugal force induced by the offset between the rotor mass centroid and its geometric axis. 
(3) The deflection torque caused by the deviation between the rotor mass centroid and its sphere centroid.

4.1. Bearing Dynamic Reaction Force. The force system consisting of dynamic reaction forces, inertia forces, and unbalance mass moments is simplified. If there is an unbalanced mass moment in the counterweight plane of the rotor part A, the mechanical equations of the five control channels can be obtained as follows based on the $\mathrm{D}^{\prime}$ Alembert principle $[12,15]$ :

$$
\left\{\begin{array}{l}
f_{a x}+f_{g x}+f_{\text {agax }}=0, \\
f_{a y}+f_{g y}+f_{\text {agay }}=0, \\
f_{a z}=0, \\
2 f_{l} R+M_{g x}-f_{\text {agay }} l_{\text {an }}=0, \\
2 f_{l} R+M_{g y}+f_{\text {agax }} l_{\text {an }}=0,
\end{array}\right.
$$

where $f_{a x}, f_{a y}$, and $f_{a z}$ represent the dynamic reaction forces in $x$-, $y$-, and $z$-axis and $f_{g x}$ and $f_{g y}$ are the components of inertia force in the $x$-and $y$-axis. $f_{\text {agax }}$ and $f_{\text {agay }}$ caused by unbalanced mass moment in the $x$ - and $y$-axis are the components of inertia force. $M_{g x}$ and $M_{g y}$ are the inertial moments induced by the inertia forces acting on the $x$ - and $\mathrm{y}$-axis, respectively.

The gyro rotor is symmetrical in the inertia principal axis $Z$, and then the inertial forces $f_{g x}$ and $f_{g y}$ are zero. The bearing dynamic reaction force $\mathrm{F}_{\mathrm{a}}$ in part $A$ of gyro rotor can be described as

$$
F_{a}=\left[\begin{array}{c}
f_{a x} \\
f_{a y} \\
f_{a z}
\end{array}\right]=\frac{-1}{l_{\mathrm{an}}}\left[\begin{array}{c}
2 f_{l} R+M_{g y} \\
2 f_{l} R+M_{g x} \\
0
\end{array}\right] .
$$

Similarly, the bearing dynamic reaction force $\mathrm{F}_{\mathrm{b}}$ in part $B$ is obtained as

$$
F_{b}=\left[\begin{array}{c}
f_{b x} \\
f_{b y} \\
f_{b z}
\end{array}\right]=\frac{-1}{l_{\mathrm{bn}}}\left[\begin{array}{c}
2 f_{l} R+M_{g y} \\
2 f_{l} R+M_{g x} \\
0
\end{array}\right] .
$$

4.2. Deflection Torque and Centrifugal Force. There is an offset $\mathbf{e}=e_{x} \mathbf{i}+e_{y} \mathbf{j}+e_{z} \mathbf{k}$ between the rotor mass centroid and the rotor sphere centroid. $e_{x}, e_{y}$, and $e_{z}$ are components of deviation e along three coordinate axes. The electromagnetic forces $f_{x}, f_{y}$, and $f_{z}$ always pass through the rotor sphere centroid, which results in a deflection torque $\mathrm{P}_{\mathrm{d}}$ around the rotor mass centroid:

$$
P_{d}=2\left[\begin{array}{l}
f_{x} \sqrt{e_{y}^{2}+e_{z}^{2}} \\
f_{y} \sqrt{e_{x}^{2}+e_{z}^{2}} \\
f_{z} \sqrt{e_{x}^{2}+e_{y}^{2}}
\end{array}\right],
$$

The centrifugal force $\mathrm{F}_{\mathrm{u}}$ under high speed induced by the offset between the rotor mass centroid and the rotor rotary axis can be written as

$$
F_{u}=m \omega^{2} \sqrt{e_{x}^{2}+e_{y}^{2}}\left[\begin{array}{c}
\sin \left(\omega t+\arctan \left(\frac{e_{x}}{e_{y}}\right)\right) \\
\cos \left(\omega t+\arctan \left(\frac{e_{x}}{e_{y}}\right)\right) \\
0
\end{array}\right] .
$$

\section{Unbalanced Mass Feedforward Control and Simulation}

5.1. Controller Model. Considering the bearing dynamic reaction forces $\mathrm{F}_{\mathrm{a}}$ and $\mathrm{F}_{\mathrm{b}}$, the deflection torque $\mathrm{P}_{\mathrm{d}}$, and the centrifugal force $F_{u}$, the control block diagram of the magnetically suspended system is obtained, which is plotted with a solid line in Figure 3. The main parameters of the system are listed in Table 1 . The centrifugal force induced by the offset between the rotor mass centroid and the rotor rotary axis is in the feedback loop. The bearing dynamic reaction forces and the deflection torque are input interferences. The feedforward compensation against three interferences is plotted with the dashed line in Figure 3. $G_{f}(s), G_{p}(s)$, and $G_{u}(s)$ are, respectively, the transfer functions of the bearing dynamic reaction forces, the deflection torque, and the centrifugal force, which can be expressed as

$$
G_{f}(s)=G_{u}(s)=G_{p}(s)=-K_{i}^{-1} K_{w}^{-1},
$$

5.2. Simulation. The rotor dynamic balance grade G1 of ISO1940 is used. The rotor rated speed is $8000 \mathrm{r} / \mathrm{min}$, and the permissible eccentricity $\delta$ of the magnetically suspended system is

$$
\delta=\frac{1000}{2 \pi \times(n / 60)} \approx 1.19 \mu \mathrm{m} .
$$

The permissible unbalance mass moment $G_{m}$ in two counterweight planes is

$$
G_{m}=\frac{\mathrm{me}}{2} \approx 3.39 \mathrm{gmm} .
$$

According to the mathematical model and the control block diagram analyzed above, the feedforward suppression simulation results of three interferences are shown in Figure 4 . The unbalance mass moment of $3.39 \mathrm{gmm}$ in two counterweight planes with the phase difference of $\pi$ is used. The components of offset $\mathbf{e}$ along three coordinate axes with the same value of $60 \mu \mathrm{m}$ are adopted.

As shown in Figure 4(a), the rotor radial and axial displacement amplitudes are, respectively, reduced from $20.8 \mu \mathrm{m}$ and $31.7 \mu \mathrm{m}$ to $16.4 \mu \mathrm{m}$ and $25.5 \mu \mathrm{m}$, when the bearing dynamic reaction force is compensated. It can be 


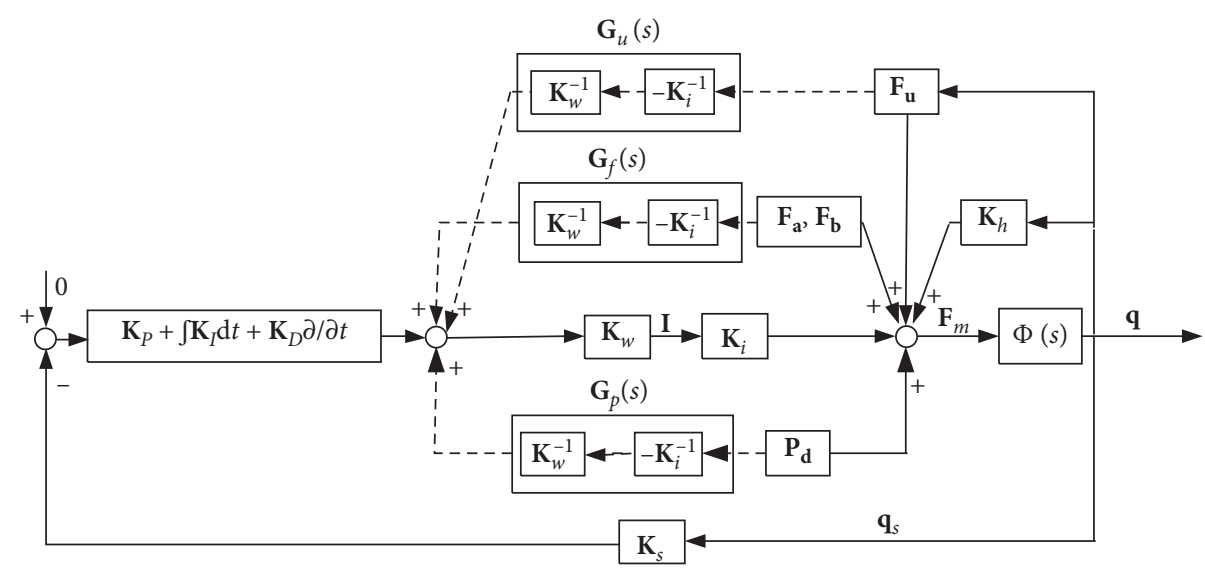

FIGURE 3: Control block diagram with feedforward compensation.

TABLE 1: The main parameters of the control system.

\begin{tabular}{lc}
\hline Parameters & Value \\
\hline$m(\mathrm{~kg})$ & 5.7 \\
$\omega\left(\mathrm{r} \cdot \mathrm{min}^{-1}\right)$ & 8000 \\
$J_{x}\left(\mathrm{~kg} \cdot \mathrm{m}^{2}\right)$ & 0.0097 \\
$J_{y}\left(\mathrm{~kg} \cdot \mathrm{m}^{2}\right)$ & 0.0097 \\
$J_{z}\left(\mathrm{~kg} \cdot \mathrm{m}^{2}\right)$ & 0.0167 \\
$\mathbf{K}_{\mathbf{i}}\left(\mathrm{N} \cdot \mathrm{A}^{-1}\right)$ & 220 \\
$\mathbf{K}_{\mathbf{h}}\left(\mathrm{N} \cdot \mathrm{m}^{-1}\right)$ & 900 \\
$\mathbf{K}_{\mathbf{s}}$ & 75 \\
$\mathbf{K}_{\mathbf{w}}$ & 0.22 \\
$\mathbf{K}_{\mathbf{P}}$ & 16 \\
$\mathbf{K}_{\mathbf{I}}$ & 130 \\
$\mathbf{K}_{\mathbf{D}}$ & 65 \\
\hline
\end{tabular}

seen in Figure 4(b) that when the feedforward suppression against deflection torque is implemented, both the displacement amplitudes are decreased to $17.2 \mu \mathrm{m}$ and $27.4 \mu \mathrm{m}$, respectively. When the feedforward suppression against the centrifugal force is only concerned, both the displacement amplitudes are reduced to $18.5 \mu \mathrm{m}$ and $29.3 \mu \mathrm{m}$, which are shown in Figure 4(c). It is obvious that feedforward suppression against three interferences plays an important role in rotor microvibration with low displacement amplitude.

The interference torques caused by the bearing dynamic reaction force and the deflection torque are about $10^{-2} \mathrm{Nm}$ and $10^{-3} \mathrm{Nm}$, respectively. Both of them are constant. The interference torque induced by the centrifugal force decreasing with the rotor vibration displacement is about $10^{-3} \mathrm{Nm}$. The loading order of three interferences has a significant effect on the rotor vibration displacement. The simulation with a loading interval between two interferences of 0.2 seconds is used, and the cosuppression effectiveness of three interferences in different loading sequences is shown in Figure 5.

The interference torque caused by the bearing dynamic reaction force is larger than the other two interferences. When the bearing dynamic reaction force is compensated firstly, the maximum ultimate vibration displacement amplitudes of $14.9 \mu \mathrm{m}$ in Figure 5(a) and $15.5 \mu \mathrm{m}$ in Figure 5(b) are relatively small, compared with the other four states. The interference torque induced by the centrifugal force is proportional to vibration displacement, which should be located behind the other two interferences. The suppression effects in Figures 5(a) and 5(c) are better than those in Figures 5(b) and 5(d), respectively. It can be seen from Figures 5(e) and 5(f) that the ultimate vibration displacements with maximum of $16.7 \mu \mathrm{m}$ and $16.9 \mu \mathrm{m}$ are relatively large. It is the mutual couplings among the three interferences that cause the difference of suppression effects under the different loading sequences. The ultimate vibration displacement in Figure 5(a) is less than the other five states, which indicates the optimal loading order is bearing dynamic reaction force, deflection torque, and centrifugal force control. The rotor vibration trajectories under the optimal loading sequence of three interferences are plotted in Figure 6 .

\section{Experiment}

The gyro rotor feedforward suppression experiment is carried out, and the MSCSG test rig is shown in Figure 7. The rotor unbalance mass moments with the phase difference of $162^{\circ}$ in two counterweight planes are, respectively, $1.43 \mathrm{gmm}$ and $1.33 \mathrm{gmm}$, after field balance experiments. The radial and axial offsets between the rotor mass centroid and the 


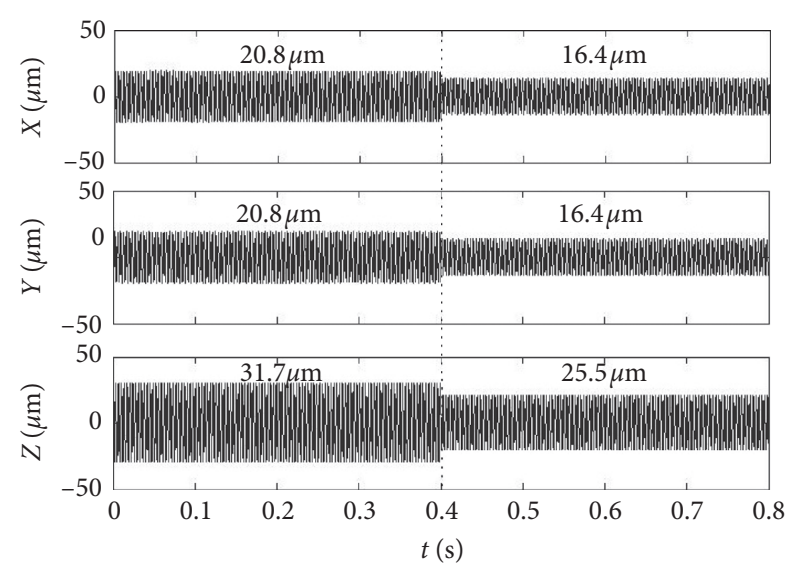

(a)

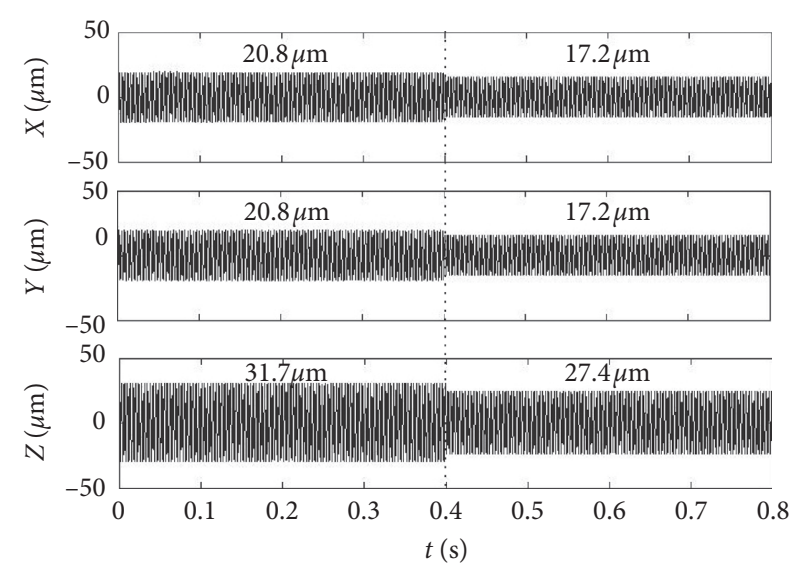

(b)

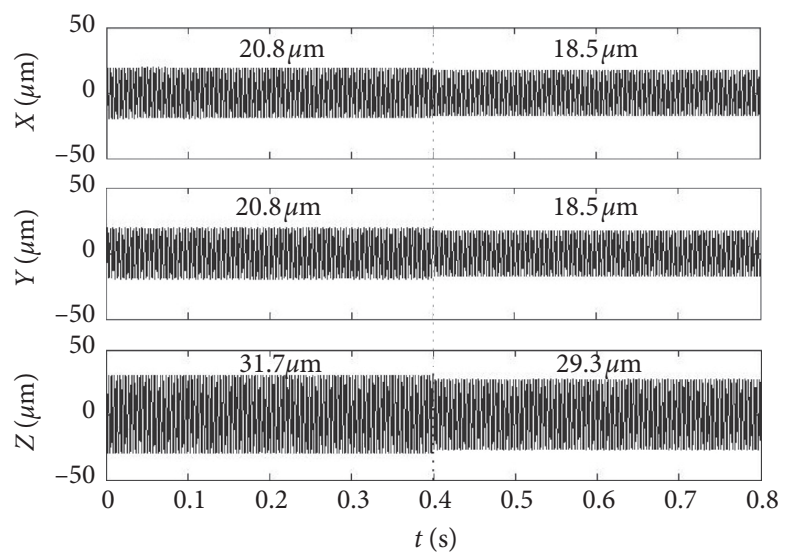

(c)

Figure 4: Feedforward suppression against three interferences: (a) bearing reaction dynamic force $\mathbf{F}_{\mathbf{a}}, \mathbf{F}_{\mathbf{b}}$, (b) deflection torque $\mathbf{P}_{\mathbf{d}}$, and (c) centrifugal force $\mathbf{F}_{\mathbf{u}}$.

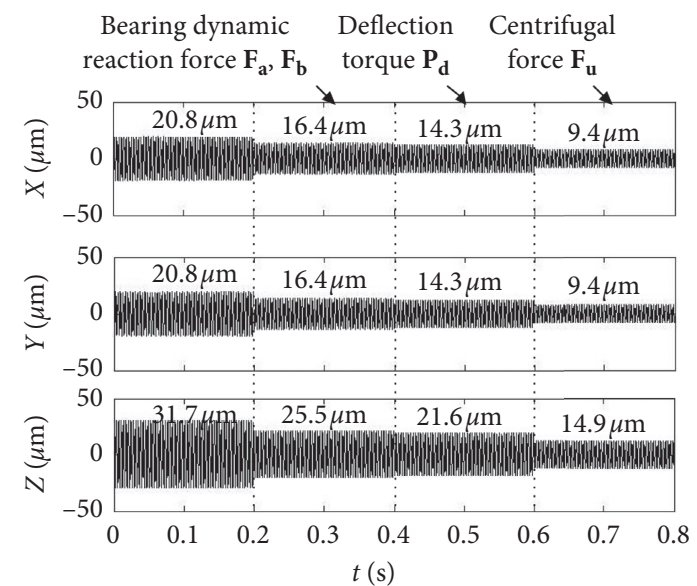

(a)

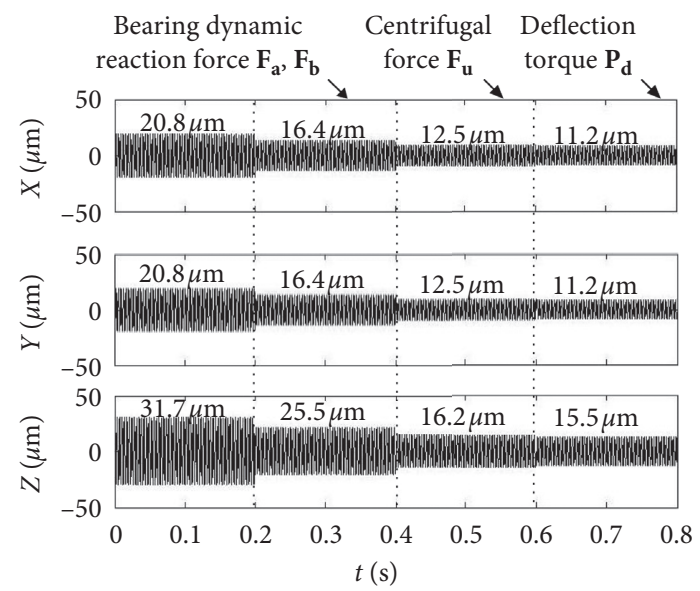

(b)

Figure 5: Continued. 


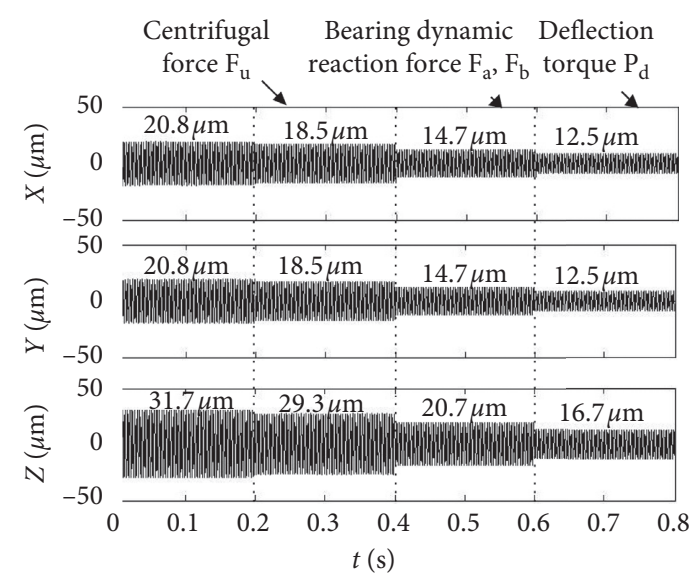

(c)

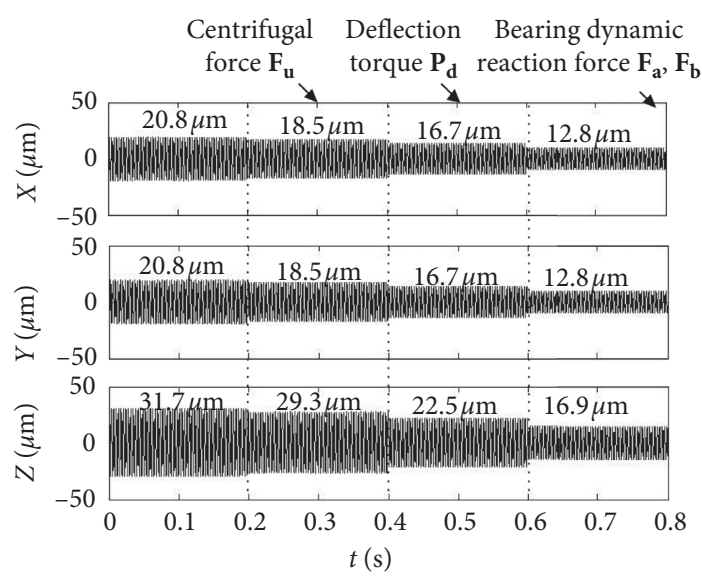

(d)

FIGURE 5: Cosuppression effectiveness of three interferences in different loading sequences.

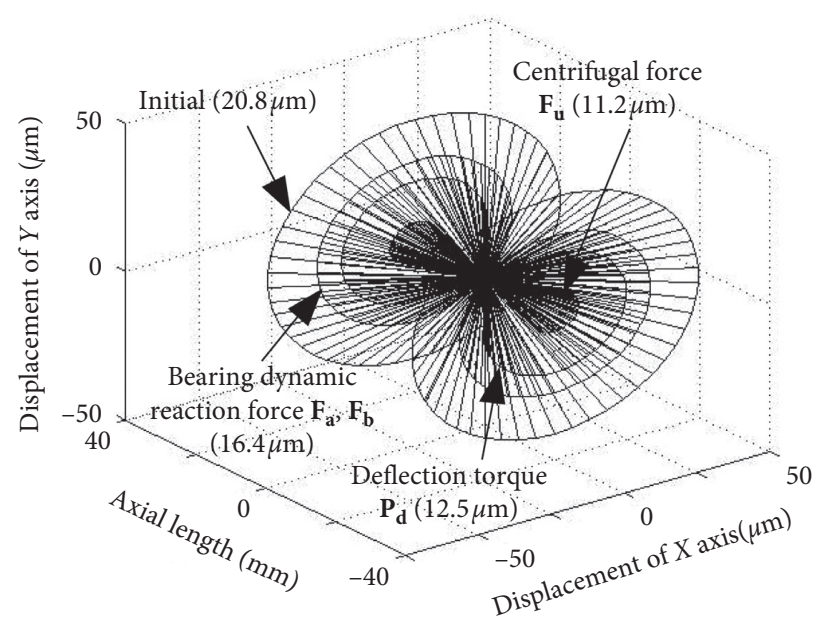

Figure 6: The rotor vibration trajectories.

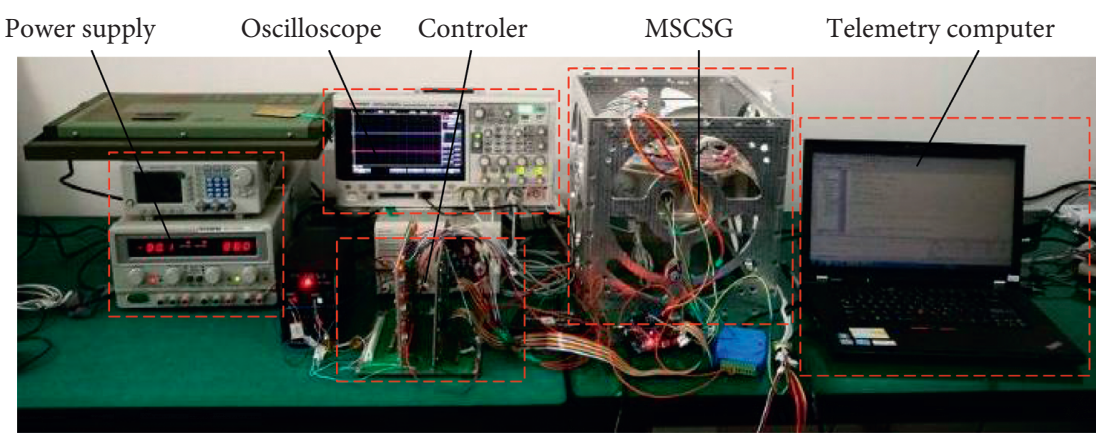

Figure 7: The MSCSG test rig.

rotor sphere centroid are about $45 \mu \mathrm{m}$ and $40 \mu \mathrm{m}$, respectively. The optimal compensation loading sequence of the bearing dynamic reaction force, the deflection torque, and the centrifugal force is used according to the simulation results above. The vibration suppression of the magnetically suspended rotor with the rated speed of $8000 \mathrm{r} / \mathrm{min}$ is implemented under the vacuum degree less than $15 \mathrm{~Pa}$. The rotor displacement is sampled by digital storage oscilloscopes, and the displacement curves are plotted in Figure 8. The radial and axial displacement amplitudes of the rotor are $19.9 \mu \mathrm{m}$ and $25.4 \mu \mathrm{m}$ before the feedforward compensation. The amplitudes are, respectively, reduced to $14.4 \mu \mathrm{m}$ and 


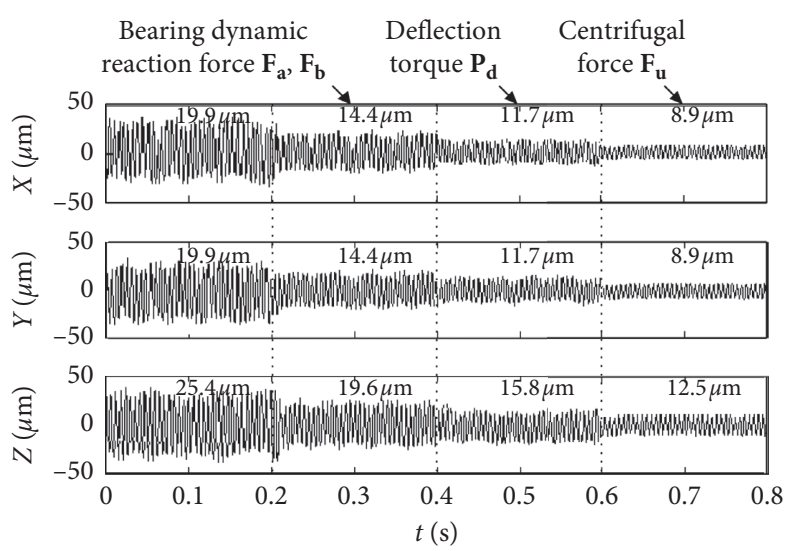

(a)

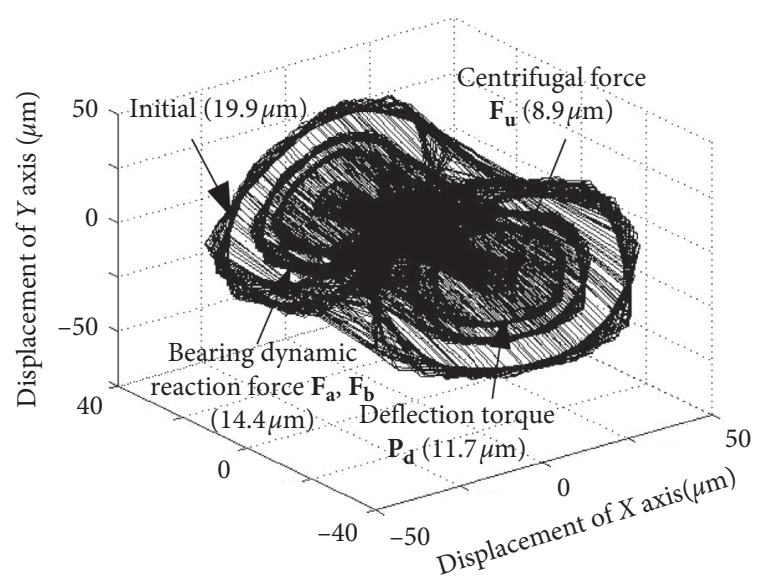

(b)

Figure 8: Experiment results with feedforward compensation: (a) the vibration displacement and (b) the rotor vibration trajectory.

$19.6 \mu \mathrm{m}$ with the responding decreasing range of $25 \%$, when the bearing dynamic reaction force compensation is loaded at the time of 0.2 seconds. The deflection torque interference is compensated at the time of 0.4 seconds, and the amplitudes are further reduced to $11.7 \mu \mathrm{m}$ and $15.8 \mu \mathrm{m}$ which is reduced by $19 \%$. The ultimate amplitudes are decreased to $8.9 \mu \mathrm{m}$ and $12.5 \mu \mathrm{m}$ on the condition that three interferences are compensated at the time of 0.6 seconds with decreasing amplitude of $22 \%$. According to the analysis above, the entire decreasing amplitude of experimental results is about $52.8 \%$, a little less than that of the simulation results about $53.7 \%$. The rotor unbalance mass moments of $1.43 \mathrm{gmm}$ and $1.33 \mathrm{gmm}$ with a phase difference of $162^{\circ}$ in two counterweight planes are asymmetry, which results in the difference between the simulation traces in Figure 6 and experimental traces in Figure 8(b).

\section{Conclusion}

In this paper, a novel MSCSG with two capabilities, attitude control and attitude measurement, is presented, and the feedforward compensation control method for the magnetically suspended spherical rotor is proposed. The magnetic bearing-rotor system dynamics model is established. Considering the three interferences, the bearing dynamic reaction force, the deflection torque, and the centrifugal force, the feedforward suppression simulations with different loading sequences are implemented. The simulation results indicate that the optimal loading sequence is the bearing dynamic reaction force, the deflection torque, and the centrifugal force. The rotor radial and axial vibration displacement amplitudes are, respectively, reduced from $20.8 \mu \mathrm{m}$ and $31.7 \mu \mathrm{m}$ to $9.4 \mu \mathrm{m}$ and $14.9 \mu \mathrm{m}$ at the speed of $8000 \mathrm{r} / \mathrm{min}$, and the corresponding decreasing amplitude are about $54.8 \%$ and $53 \%$. The prototype experimental results show that the radial and axial vibration displacement amplitudes are, respectively, suppressed from $19.9 \mu \mathrm{m}$ and $25.4 \mu \mathrm{m}$ to $8.9 \mu \mathrm{m}$ and $12.5 \mu \mathrm{m}$, and its decreasing amplitude is $55.2 \%$ and $50.8 \%$, respectively. The difference between the simulation and experiment results is caused by the deviation of the two unbalance mass moments and their phase. The experimental results are in good agreement with the simulation, which indicates the MSCSG rotor unbalance vibration is suppressed effectively by the feedforward compensation method.

\section{Data Availability}

The data used to support the findings of this study are available from the corresponding author.

\section{Conflicts of Interest}

The authors declare that there are no conflicts of interest regarding the publication of this paper.

\section{Acknowledgments}

This paper was supported by the Training Funded Project of the Beijing Youth Top-Notch Talents of China (Grant no. 2017000026833ZK22) and the Support Project of High-Level Teachers in Beijing Municipal Universities in the Period of 13th Five-Year Plan (Grant no. CIT\&TCD201804034).

\section{References}

[1] O. H. Shahmohamadi, "Active nutation control of an asymmetric spacecraft using an axial reaction wheel," $A d$ vances in Space Research, vol. 58, no. 11, pp. 2287-2303, 2016.

[2] H. Wang, Q. Han, R. Luo, and T. Qing, "Dynamic modeling of moment wheel assemblies with nonlinear rolling bearing supports," Journal of Sound and Vibration, vol. 406, pp. 124-145, 2017.

[3] Q. Liu, K. Wang, Y. Ren, X. C. Chen, and Y. Zhao, "Optimization design of launch locking protective device (LLPD) based on carbon fibber bracket for magnetically suspended flywheel (MSFW)," Acta Astronautica, vol. 154, pp. 9-17, 2019.

[4] C. Peng, S. Zheng, Z. Huang, and X. Zhou, "Complete synchronous vibration suppression for a variable-speed magnetically suspended flywheel using phase lead compensation," 
IEEE Transactions on Industrial Electronics, vol. 65, no. 7, pp. 5837-5846, 2018.

[5] Y. Liu, S. S. Ming, S. Y. Zhao, J. Y. Han, and Y. X. Ma, "Research on automatic balance control of active magnetic bearing-rigid rotor system," Shock and Vibration, vol. 2019, Article ID 3094215, 13 pages, 2019.

[6] M. Privat and A. D. Costa, "Pneumatic locking device for magnetic bearing reaction wheel," in Proceedings of the 10th European Space Mechanisms and Tribology Symposium, San Sebastian, Spain, September 2003.

[7] P. Anstett, M. Souliac, C. Rouyer, and M. Gauthier, "SPOT: the very first satellite to use magnetic bearing wheels," in Proceedings of the 33rd Congress of the International Astronautical Federation, Paris, France, September 1982.

[8] K. McLallin, J. Fausz, and R. Jansen, "Aerospace flywheel technology development for IPACS applications," in Proceedings of the IECEC'01: 36th Intersociety Energy Conversion Engineering Conference, IECEC2001-AT-82, Savannah, GA, USA, July 2001.

[9] G. F. Xu, Y. W. Cai, Y. Ren, C. J. Xin, and Q. Liu, “Application of a new Lorentz force-type magnetic bearing in tilting control for magnetically suspended control \& sensitive gyroscope with cross sliding model control," Transactions of the Japan Society for Aeronautical and Space Sciences, vol. 59, no. 1, pp. 107-109, 2016.

[10] C. J. Xin, Y. W. Cai, Y. Ren, and Y. H. Fan, “Compensation on-line of errors caused by rotor centrifugal deformation for a magnetically suspended sensitive gyroscope," Journal of Electrical Engineering \& Technology, vol. 13, no. 2, pp. 10301041, 2018.

[11] Q. Liu, H. Li, W. Wang, C. Peng, and Z. Yin, "Analysis and experiment of 5-DOF decoupled spherical vernier-gimballing magnetically suspended flywheel (VGMSFW)," IEEE Access, vol. 8, pp. 111707-111717, 2020.

[12] C. Peng, K. W. Cai, Z. Q. Deng, and K. X. Li, "Vibration torque suppression for magnetically suspended flywheel using improved synchronous rotating frame transformation," Shock and Vibration, vol. 2019, Article ID 3607164, 13 pages, 2019.

[13] D. X. Wang, N. X. Wang, K. S. Chen, and C. Ye, "Dynamic characteristics of magnetic suspended dual-rotor system by Riccati transfer matrix method," Shock and Vibration, vol. 2019, Article ID 9843732, 22 pages, 2019.

[14] M. Takeshi, "Analysis on the fundamental properties of active magnetic bearing control system by a transfer function approach," The Japan Society of Mechanical Engineers, vol. 44, no. 2, pp. 367-373, 2001.

[15] J. Fang, X. Xu, J. Tang, and H. Liu, “Adaptive complete suppression of imbalance vibration in AMB systems using gain phase modifier," Journal of Sound and Vibration, vol. 332, no. 24, pp. 6203-6215, 2013.

[16] K. J. Jiang, C. S. Zhu, and M. Tang, "A uniform control method for imbalance compensation and automation balancing in active magnetic bearing-rotor systems," Journal of Dynamic Systems, Measurement, and Control, vol. 134, no. 2, Article ID 021006, 2012.

[17] J. Shi, R. Zmood, and L. Qin, "Synchronous disturbance attenuation in magnetic bearing systems using adaptive compensating signals," Control Engineering Practice, vol. 12, no. 3, pp. 283-290, 2004.

[18] F. Betschon and C. R. Knospe, "Reducing magnetic bearing currents via gain scheduled adaptive control," IEEE/ASME Transactions on Mechatronics, vol. 6, no. 4, pp. 437-443, 2001.

[19] H. Gao, "Real-time feed-forward force compensation for active magnetic bearings system based on $H_{\infty}$ controller,"
Chinese Journal of Mechanical Engineering, vol. 24, no. 1, pp. 58-66, 2011.

[20] T. Wei and M. Xiang, "Autobalancing of high-speed rotor suspended by magnetic bearings using LMS adaptive feedforward compensation," Journal of Sound and Vibration, vol. 20, no. 9, pp. 1428-1436, 2014.

[21] S. Zheng and R. Feng, "Feedforward compensation control of rotor imbalance for high-speed magnetically suspended centrifugal compressors using a novel adaptive notch filter," Journal of Sound and Vibration, vol. 366, pp. 1-14, 2016.

[22] J. Q. Tang, B. Liu, J. C. Fang, and S. S. Ge, "Suppression of vibration caused by residual unbalance of rotor for magnetically suspended flywheel," Journal of Sound and Vibration, vol. 19, no. 13, pp. 1962-1979, 2005.

[23] X. Xu, S. Chen, and Y. Zhang, "Automatic balancing of AMB systems using plural notch filter and adaptive synchronous compensation," Journal of Sound and Vibration, vol. 374, pp. 29-42, 2016.

[24] Q. Chen, G. Liu, and S. Zheng, "Suppression of imbalance vibration for AMBs controlled driveline system using doubleloop structure," Journal of Sound and Vibration, vol. 337, pp. 1-13, 2015.

[25] L. Tang and Y. Q. Chen, "Model development and adaptive imbalance vibration control of magnetic suspended system," Acta Astronautica, vol. 65, no. 9-10, pp. 1506-1514, 2009.

[26] C. Bi, D. Z. Wu, Q. Jiang, and Z. J. Liu, "Runout compensation in active magnetic bearings with iterative learning control scheme," in Proceedings of the Asia-Pacific Magnetic Recording Conference, Seoul, South Korea, August 2004.

[27] L. Li, T. Shinshi, C. Iijima, X. Zhang, and A. Shimokohbe, "Compensation of rotor imbalance for precision rotation of a planar magnetic bearing rotor," Precision Engineering, vol. 27, no. 2, pp. 140-150, 2003.

[28] T. R. Grochmal and A. F. Lynch, "Precision tracking of a rotating shaft with magnetic bearings by nonlinear decoupled disturbance observers," IEEE Transactions on Control Systems Technology, vol. 15, no. 6, pp. 1112-1121, 2007.

[29] P. C. Tung, M. T. Tsai, K. Y. Chen, Y. H. Fan, and F. C. Chou, "Design of model-based unbalance compensator with fuzzy gain tuning mechanism for an active magnetic bearing system," Expert Systems with Applications, vol. 38, no. 11, pp. 12861-12868, 2011.

[30] B. Gerlach, M. Ehinger, H. K. Raue, and R. Seiler, "Digital controller for a gimballing magnetic bearing reaction wheel," in Proceedings of the AIAA Guidance, Navigation, and Control Conference and Exhibit, San Francisco, CA, USA, August 2005.

[31] C. Hui, L. Shi, J. Wang, and S. Y. Yu, "Adaptive unbalance vibration control of active magnetic bearing systems for the HTR-10GT," in Proceedings of the 18th International Conference on Nuclear Engineering, Xi'an, China, May 2010.

[32] S. Zhou and J. Shi, "Imbalance estimation for speed-varying rigid rotors using time-varying observer," Journal of Dynamic Systems, Measurement, and Control, vol. 123, no. 4, pp. 637-644, 2001.

[33] B. Xiang and J. Tang, "Suspension and titling of verniergimballing magnetically suspended flywheel with conical magnetic bearing and Lorentz magnetic bearing," Mechatronics, vol. 28, pp. 46-54, 2015. 